\title{
O ENSINO DE FARMACOLOGIA MÉDICA DIRECIONADO À REALIDADE: EXEMPLO DE UMA EXPERIÉNCIA PIONEIRA
}

\author{
Flávio Danni Fuchs* \\ Lenita Wannmacher** \\ Marcelo Victor ${ }^{* * *}$
}

\section{Resumo}

Os autores preconizam o ensino de Farmacologia Médica voltado à realidade de prescrição em nosso meio, relatando experiências pedagógicas fundamentadas na solução de problemas. Dentre elas, que incluem solução de casos clínico-farmacológicos simulados, seminários de análise crítica da literatura original em Farmacologia aplicada e aulas expositivas, salienta-se a atividade prática da disciplina: preenchimento de fichas de avaliação da prescrição medicamentosa de pacientes internados no hospital-escola. No presente trabalho descrevem-se a metodologia dessa atividade e os resultados obtidos.

\section{Introdução}

Os jovens que ingressam no curso médico estão, em geral, fortemente motivados para o exercício profissional, desejando precocemente responder aos questionamentos sobre saúde e doença.

Em atendimento a essas aspiraçőes, o ensino, desde o início do curso, deve preocupar-se com a formaçäo global do aluno estimulando a capacidade de transferir os principios cientfficos e metodológicos às situaçōes clínicas que exijam a aplicação dos mesmos, de acordo com as necessidades prevalentes do meio onde o aluno se inserirá como profissional.

Dentro deste contexto, o ensino de Farmacologia Médica na Universidade Federal do Rio Grande do Sul, desde 1978, passou a centrar-se na abordagem de solução de problemas, proporcionando o embasamento farmacológico, cientffico e racional à escolha e à administração de fármacos essenciais que servem ao manejo medicamentoso das doenças prevalentes. Uma descrição compreensiva das mudanças e a avaliação dos resultados de 5 anos de implementação foram anteriormente publicadas ${ }^{3}$.

Fundamentalmente, 0 aprendizado teve seu eixo deslocado da farmacodinâmica clássica para o estudo da eficácia e efetividade de fármacos em seres humanos e, assim, o ensino prático também se deslocou da visualização do efeito farmacológico em animal de experimentação para a avaliação de uma dada prescrição medicamentosa em um paciente.

\footnotetext{
- Professor Adjunto do Departamento de Fisiologia, Farmacologia e Bioffsica da UFRGS e do Departamento de Ciências Fisiológicas da FFFCMPA.

** Professor Adjunto do Departamento de Fisiologia, Famacologia e Biofísica da UFRGS.

**A Acadêmico de Medicina da UFRGS.
}

Dentre as principais atividades desenvolvidas na disciplina estão a solução de casos clínico-farmacológicos constantes de um livro de exercícios ${ }^{2}$; a realização de um simpósio sobre um tema central onde, a partir de artigos originais, são discutidos os métodos e os resultados da investigação farmacológica aplicada, com o intuito de estimular a atualização constante e o senso crftico; o preenchimento e discussão de ficha de avaliação farmacológica (FAF), transcrita na figura 1, realizada individualmente pelo aluno junto a pacientes internados no hospital-escola e por eles acompanhados na disciplina de Medicina Interna.

Essa última modalidade pode ser encarada como a atividade prática da disciplina e objetiva proporcionar ao aluno uma visão reallstica das implicações da prescrição medicamentosa. Através da FAF são sopesados os fatores que levam à escolha de um dado fármaco, definidos os padrões de administração e mensurados os efeitos benéficos e riscos.

Os alunos preenchem de 4 a 6 fichas durante o semestre, sob orientação e supervisão dos professores e monitores da disciplina, a partir da prescrição feita por médicos residentes e assistentes a pacientes internados nos hospitais universitários.

$\mathrm{Na}$ avaliaçāo da disciplina acima citada ${ }^{3}$, constatouse que a FAF era uma modalidade de ensino cotada pelos alunos como muito boa ou boa $(78 \%)$, contribuindo de forma muito significativa ( $72 \%$ ) para o aprendizado e na qual eles se autoavaliaram como tendo participação $(87 \%)$, aproveitamento $(84,2 \%)$ e envolvimento $(82,6 \%)$.

No último semestre de 1987, realizou-se uma enquete entre os 64 alunos da disciplina sobre as atividades implementadas. No que diz respeito à FAF, 46 alunos $(71,8 \%)$ opinaram pela manutenção da atividade sem alteraçōes e $18(28,2 \%)$ postularam modificaçōes quanto à operacionalizaçāo e não quanto ao mérito. Nenhum deixou de participar da atividade ou opinou pela supressão da FAF.

Como exemplo do nivel de elaboração mental, senso crítico e capacidade de transferência de conhecimentos que se pode atingir com esse aprendizado, transcreve-se aqui, na integra, a FAF elaborada por um aluno ( $6^{\circ}$ semestre do curso médico), apresentada à disciplina no final de 1987.

\section{Exemplo de uma Ficha de Avaliação Farmacologica}

\section{Fármaco: PROPRANOLOL}

Identificação do paciente: J.A.S., 60 anos, masculino, bran$\mathrm{co}$, natural de Bagé, procedente de Porto Alegre, mecânico de automóveis, leito 717A, prontuário n.... 
Diagnósticos: Hemorragia digestiva alta devido a sangramento de varizes esofágicas, hipertensão porta, cirrose hepática.

\section{Indicação: incorreta}

O paciente é portador de hepatopatia crônica e apresenta varizes no esôfago há um ano aproximadamente. Não é alcoólatra e é HbsAg -. Baixou em 12.11 .87 pelo $5^{\circ}$ episódio de sangramento das varizes no corrente ano.

A literatura (na pequena revisão por nós efetuada) não autoriza o uso de propranolol no presente caso. Bem estabelecido está que a droga é capaz de reduzir significativamente e de forma sustentada a pressão no leito venoso portal. Doses de 2 mg. $\mathrm{kg}^{-1}$, ou capazes de reduzir a freqüência cardíaca ( $F C$ ) em $25 \%$, diminuem em $5 \mathrm{~mm} \mathrm{Hg}$ a pressão portal. Esse efeito farmacodinâmico aparentemente benéfico e devido à diminuição do débito cardíaco e a uma ação de relaxamento local na parede vascular, não foi efetivo em alguns ensaios realizados no sentido de prolongar a sobrevida e reduzir os sangramentos.

Aparentemente o uso é indicado em pacientes com varizes diagnosticadas, mas que ainda não sangraram e em pacientes alcoólatras que já sofreram hemorragia, mas sobreviveram e estão compensados da cirrose, isto é, não têm icterícia, ascite ou encefalopatia. O fármaco nảo é eficaz em pacientes descompensados e, inclusive, pode piorar o quadro por indução de encefalopatia e por piora do débito cardlaco no evento de sangramento, pelo bloqueio da resposta taquicárdica necessária à manutenção do débito. Isto pode ser fatal se 0 atendimento tardar, pela precipitação de choque hemorrágico mais rapidamente. Pacientes com ascite podem depender do sistema renina-angiotensina para manter uma pressão arterial e o propranolol pode causar um colapso circulatório por bloqueio da secreção renal de renina.

Este paciente não estã nos grupos em que o fármaco parece ser efetivo porque já sofreu vários sangramentos $\mathrm{e}$ porque está descompensado da cirrose, com ascite há três meses e encefalopatia leve crônica, caracterizada por um tênue asterixis. O fármoco não deve ser usado com esta indicação a não ser em estudos. Indicação incorreta, visto não haver evidência que comprove sua utilidade no quadro apresentado pelo paciente.

Esquema de administração: $1 / 2$ comprimido de $40 \mathrm{mg}$ por V.O. de $12 / 12$ horas.

A via de administração está correta, havendo boa absorçäo, apesar do metabolismo intenso de $1^{\text {a }}$ passagem pelo fígado. $O$ intervalo está acertado também, de acordo com a meia-vida de \pm 5 horas. Existe, entretanto, uma alta variabilidade nas concentrações no plasma (até 20 vezes). Desta forma o ajuste de dose deverá ser feito por FC diminuída em $25 \%$, o que garantiria uma queda efetiva da pressão portal, se fosse de fato correta a indicaçăo. Deveríamos, então, ir aumentando gradualmente esta dose inicial até obtermos o efeito supostamente terapêutico.

Não são necessários cuidados com a alimentação ou horário e a forma farmacêutica é adequada.

Efeitos positivos: A não ocorrência de sangramento numa evolução de 4 dias não é um dado positivo na avaliação do fármaco, visto haver pouca possibilidade de que este efeito seja devido ao seu uso. Existem trabalhos mostrando que uma queda na $\mathrm{FC}$ em $25 \%$ corresponde a uma queda de 5 $\mathrm{mm}$ HG na pressão venosa portal, logo este é um dado melhor para avaliar o efeito farmacológico do propranolol.
Um dia antes do início do uso, a FC era de $86 \mathrm{bpm}$. No quarto dia de uso a FC era de 84 bpm. Năo há como avaliar se esta pequena variaçäo é significativa, mas provavelmente não é. Como este é o parâmetro mais adequado, não há evidência de nenhum efeito positivo. O propranolol é uma droga que tem uma variação de até 20 vezes na concentração plasmática depois de doses iguais em individuos diferentes e isto, junto com o pouco tempo de uso, justifica a ausência de efeitos.

\section{Efeitos adversos}

Não se observou nenhum enquanto o paciente esteve internado (4 dias). No momento, aguarda leito para internação por encefalopatia hepática. Há trabalhos correlacionando o uso indevido de propranolol com tal fato, em casos de pacientes com varizes esofágicas e hipertensão porta, de forma que essa reaçāo adversa tem uma possibilidade de causa-efeito duvidosa, mas existente. Obviamente este é um evento esperado num cirrótico terminal, mas ele nos lembra da necessidade da justa adequação das indicaçōes farmacológicas em nossos pacientes.

\section{Fármacos simultâneos}

Metoclopramida 1 ampola EV de $6 / 6$ horas se necessário para náuseas ou vômitos;

Acetaminofem $500 \mathrm{mg}$, VO de $6 / 6$ horas se necessário para dor ou febre;

Cefoxitina $1 \mathrm{~g}$ EV de $6 / 6$ horas;

Bórax com hortelã para bochecho 4 vezes ao dia.

Não há interação descrita entre os fármacos citados e o propranolol.

Início: $16 / 11 / 87$

Término: $25 / 11 / 87$

\section{Bibliografia}

1. BERK JE. Bockus Gastroenterology. 4 ed., Philadelphia, WB Saunders, 1985. v.5, cap. 159.

2. BLEEDING OESOPHAGEAL VARICES. The Lancet, 1: 139-41, 1984.

3. BURROUGHS AK et allii. A control trial of propranolol for the prevention of recurrent variceal hemorrhage in patients with cirrhosis. The New England Journal of Medicine, 309: 1539-42, 1983.

4. GILMAN AG, GOODMAN LS, RALL TW, MURAD F (eds). Goodman and Gilman's The Pharmacological Basis of Therapeutics. 7 ed., New York, McMillan, 1985.

5. LEBREC D; NOVEL O; CORBIC M; BENHAMOU J. Propranolol: a medical treatment for portal hypertension? The Lancet, 2: 180-82, 1980.

6. PASCAL JP, CALES $P$ and a MULTICENTER STUDY GROUP: Propranolol in the prevention of first upper gastrointestinal tract hemorrhage in patients with cirrhousis of the liver and esophageal varices. The New England Journal of Medicine, 317: 856-61, 1987.

7. SOGGARD PE. Propranolol in portal hypertension. The Lancet, 1: 1204, 1981

8. WYNGAARDEN JB, LLOYD HS (eds). Cecil Textbook Medicine. 17 ed., Japan, Igaku-Shoin Saunders Edition, 1985. 


\section{Comentários}

A FAF apresentada ilustra claramente 0 atingimento dos objetivos propostos por essa atividade. Parece evidente que sua publicaçăo sob o título "O uso de propranolol na prevenção de sangramento em pacientes com hipertensão porta" constituiria uma excelente revisão sobre o tema.

O leitor pode questionar se não é essa uma exceção, devida à diferenciação, também excepcional, do estudante. De fato, essa FAF se salienta pelo domínio de linguagem de quem a fez e pela controvérsia do tema abordado, mas a grande maioria dos alunos apresenta fichas semelhantes ao exemplo, no tocante à discussão do mérito dos diferentes aspectos envolvidos na prescrição medicamentosa.

Isso se acentua ao longo da disciplina, já pelas vivências acumuladas pelo aluno em Farmacologia e Medicina Interna, já pela familiarização com o método, já pela orientação de pequenos grupos (6-7 alunos), que os professores e monitores fazem no âmbito do próprio hospital.

É importante salientar que a ética da avaliação crítica é uma premissa e que, no decorrer dos dez anos de implementação deste trabalho, nunca se gerou qualquer queixa por parte de quem prescreve.

Em conclusão, julga-se ser essa uma forma válida de realizar ensino prático em Farmacologia, concorrendo para que 0 aluno se aproprie dos critérios farmacológicos que suportam um emprego mais adequado dos medicamentos.

Por outro lado, a FAF constitui-se na operacionalização da integração entre os ciclos básicos e clínicos do curso médico, integração essa que, segundo Fraga Filho ${ }^{1}$, "não deve ser mais discutida, mas sim posta em prática, através de diferentes modalidades, a partir da disposição para o processo de mudança da escola médica".

\section{Summary}

The authors preconize that the teaching of Medical Pharmacology should be oriented to the most common drugs employed in Brazil and describe the experience of a discipline based on problem solving. Beside other didactic procedures used, as seminars for discussion of simulated clinical-pharmacological cases, journal clubs with original articles on applied Pharmacology, and lectures, it is pointed out in this communication the methodology and the results of the practical classes of the discipline: filling of the Pharmacological Evaluation Card, which analyzes the drug prescription of inpatients of the University affiliated hospital.

\section{Referências Bibliográficas}

1. FRAGA FILHO, C. Integração dos ciclos básico e profissional: visão do clinico. In: CONGRESSO BRASILEIRO DE EDUCAÇÃO MÉDICA, 22 Anais do XXII Congresso Brasileiro de Educação Médica. Uberlândia, 1985. p.83-8.

2. FUCHS, F.D. \& WANNMACHER, L. Farmacologia Médica: Abordagem de Solução de Problemas, 3 ed., Porto Alegre, Editora da Universidade, 1986.

3. WANNMACHER, L; FUCHS, F.D.; FERREIRA, M.B. O ensino de Farmacologia Médica Aplicada: uma revisão de 5 anos. Ciência e Cultura, 38: 481-8, 1986.

Endereço do autor.

Setor de Farmacologia

Instituto de Biociências/UFRGS

Av. Sarmento Leite, 500 s. 202

90040 Porto Alegre RS

\section{FICHA DE AVALIAÇÃO FARMACOLÓGICA}

\section{IDENTIFICAÇÃO DO PACIENTE}

FARMACO:

Nome:

Natural:

Peso:

Leito:

DIAGNÓSTICOS

INDICAÇÃO:

ESQUEMA DE DOSAGEM:

EFEITOS POSITIVOS:

EFEITOS ADVERSOS:

OUTROS FÁRMACOS EM USO:

BIBLIOGRAFIA:

INICIO DA OBSERVAÇÃO

TÉRMINO DA OBSERVAÇÃO:
Idade: Sexo: Cor:

Endereço:

Altura:

Registro:

Figura 1. Estrutura da Ficha de Avaliaçáo Farmacológica

ESTUDANTE:

INSTRUTOR: 